Doi: https://doi.org/10.17398/2340-4256.14.475

\title{
APORTACIONES DE LA GEROTRASCEDENCIA EN EL ENVEJECIMIENTO: UNA VISIÓN CENTRADA EN LA ÉTICA DE NECESIDADES
}

\section{CONTRIBUTIONS OF GEROTRASCEDENCE IN AGING: A VISION FOCUSED ON THE ETHICS OF NEEDS}

\author{
ANTONIO SÁNCHEZ CABACO \\ Universidad Pontificia de Salamanca \\ LUZ Ma FERNÁNDEZ MATEOS \\ Universidad Pontificia de Salamanca
}

Recibido: 04/12/2018 Aceptado: 23/01/2019

\section{RESUMEN}

En este artículo se desarrolla una formulación operativa de la teoría de la gerotranscendencia de Tornstam $(1989,2000,2011)$ para justificar las intervenciones en el adulto mayor centradas en la ética de necesidades, a diferencia de la óptica tradicional basada en los cuidados. Partiendo de una justificación sobre la vulnerabilidad de esta etapa del ciclo vital en claves emocionales y de soledad, se delimita la cuestión del sentido vital como elemento fundamental de la teoría. A continuación, se desarrolla una propuesta metodológica basada en la Cognición Instruccional Positiva para terminar argumentando la finalidad de la misma y los resultados que esta nueva alternativa supone en el campo del envejecimiento. Además, el abordaje se realiza desde un nuevo marco explicativo, donde el sufrimiento, a diferencia de los sistemas nosológicos clínicos (DSM-V o CIE-11) es reinterpretado desde la percepción de poder, significado, amenaza y respuestas a las mismas. Un análisis, en definitiva, que abre perspectivas para abordar el 
reto del envejecimiento desde enfoques psicosociales acordes a la radiografía sociodemográfica del siglo XXI.

Palabras clave: Gerotranscendencia, Adultos Mayores, Envejecimiento Positivo.

\section{ABSTRACT}

In this article, an operative formulation of Tornstam's gerotranscendancy theory $(1989,2000,2011)$ is developed to justify interventions in the elderly focused on the ethics of needs, unlike the traditional optics based on care. Starting from a justification about the vulnerability of this stage of the life cycle in emotional keys and of solitude, the question of the vital sense is delimited as a fundamental element of the theory. Then a methodological proposal based on Positive Instructional Cognition is developed to finish arguing the purpose of the same and the results that this new alternative supposes in the field of aging. In addition, the approach is made from a new explanatory framework, where suffering, unlike clinical nosological systems (DSM-V or CIE-11) is reinterpreted from the perception of power, meaning, threat and responses to them. An analysis, in short, that opens perspectives to tackle the aging challenge from psychosocial approaches according to the sociodemographic radiography of the 21 st century.

Keywords: Gerotranscendence, Older Adults, Positive Aging.

\section{INTRODUCCIÓN}

La realidad sociodemográfica actual de las sociedades occidentales desarrolladas, y próximamente para las que están en vías de desarrollo, marcan como hoja de ruta prioritaria la atención integral al adulto mayor. Como señala la geriatra italiana Daniela Mari (2018) este nuevo escenario del envejecimiento de las sociedades plantea una paradoja, dado que el logro utópico de las tasas de longevidad actuales podría destrozar la economía de algunos países por los costos asociados al mantenimiento de los sistemas de salud. Además de las cifras y sus consecuencias, de cara a la planificación y la articulación de políticas preventivas y de atención a corto, medio y largo plazo (OMS, 2015), debe articularse un programa de ingeniería social para comprometer a la sociedad en su conjunto. Porque el objetivo último de este proceso debe combinar lo cuantitativo (años a la vida) con lo cualitativo (vida a los años). Lo que queremos subrayar en este artículo es que un planteamiento que haga viable el lema de "una sociedad para todas las edades" debe contemplar esta última etapa del ciclo vital con visiones científicas no reduccionistas que aboguen por abordajes humanistas y psicosociales en sus visiones y propuestas aplicadas (Cabaco, 2016). 
Como se ha señalado reiteradamente en la tradición geriátrica y gerontológica el proceso de envejecimiento no sólo se limita al deterioro de células y genes, sino que hay posibilidades de aprendizaje y plasticidad hasta periodos avanzados de la vida (Calero \& Galiano, 2009). Y aunque las distintas teorías biológicas del envejecimiento han identificado múltiples factores y explicaciones del mismo (papel del acortamiento de los telómeros, inflamaciones que afectan al sistema inmunológico, oxidación por los radicales libres, etc.) no contamos con una visión integradora de por qué la pérdida de homeostasis conduce al envejecimiento y la muerte, aunque sí se han identificado factores de protección (Snowdon, 2003). Ciertamente tenemos que conocer los procesos que subyacen para poder aplicar las mejores estrategias de tratamiento, y no sólo farmacológicas, que optimizan la calidad de vida y el bienestar en todos los planos de la realidad del adulto mayor.

Nuestra experiencia, de más de dos décadas en este campo, nos lleva a reconocer un sesgo de planeamiento dado que admitiendo las importantes implicaciones biológicas del proceso, nos limitaremos a desarrollar la justificación primero, la delimitación después y la metodología y finalidad, por último, de las terapias no farmacológicas (TFN) en el envejecimiento saludable, activo o positivo (Cabaco \& Fernández-Mateos, 2014). Se va a responder a los cuatro interrogantes de un proceso de investigación intentando clarificar en primer lugar el por qué, incidiendo en dos claves: implicaciones de la longevidad y radiografía de la soledad y depresión en el adulto mayor sin redes psicosociales. El segundo apartado se centrará en la delimitación de los aspectos centrales de la age identity, la respuesta al qué, en el que además de las dimensiones psico-sociales se integra la espiritualidad, es decir, la denominada gerotranscendencia. En tercer lugar, se abordará la respuesta al cómo, es decir, qué abordaje metodológico es posible para conseguir estos objetivos y, además, que haya conseguido obtener resultados basados en la evidencia científica. Por último, se intentará responder al por qué, es decir, la finalidad que estas intervenciones (TFN) persiguen de la promoción de salud y la autonomía del adulto mayor. La felicidad es una meta en cualquier momento del ciclo vital y, por lo tanto, también un quehacer y responsabilidad de los científicos facilitar caminos para su logro. Así, entendemos que el paradigma bio-psico-social-espiritual se plasma no sólo en concepciones teorizantes sino que se vislumbra para la praxis profesional.

Es necesario visualizar en las representaciones sociales el nuevo rol del adulto mayor (el 1 de octubre se celebra el día Internacional de las Personas Mayores) pero no basta. Los colectivos profesionales, como el ejemplo del COP (Colegio Oficial de Psicólogos), promueven medidas específicas para avanzar en este camino de envejecer saludablemente. Y, además, para afrontar también 
las situaciones de patologías, cronicidad o los malos tratos a las personas mayores que son realidades también necesarias de contemplar y generar alternativas optimizadoras centradas en el buen trato (Pérez-Rojo \& Chullan, 2016).

\section{JUSTIFICACIÓN: LA RESPUESTA AL POR QUÉ DE LA VULNERABI- LIDAD}

La respuesta a este primer interrogante se podría articular considerando tres niveles justificativos que están estrechamente relacionados: un aumento poblacional de adultos mayores, que viven cada vez en una proporción mayor situaciones de soledad y que esta circunstancia acarrea mayor vulnerabilidad a la depresión. La primera clave es el logro social y sanitario que deriva en un aumento de la longevidad media, ya que el máximo es un techo que como cualquier especie no podemos superar. Y esta realidad traducida en datos implica que en Europa desde la década de los 60 se duplica cada diez años el número de centenarios. Dado que la longevidad no siempre es saludable este cambio implica redefinir los sistemas de atención a corto y medio plazo, de modificar la prioridad en la atención y priorizar las políticas preventivas (OMS, 2015).

Sin embargo, son alarmantes las cifras de la realidad de la convivencia de muchos mayores en situaciones de extremo aislamiento y soledad, sin redes de apoyo familiar o social y con casos reiterados de mortalidad descubiertos meses o años después del acontecimiento. Esta epidemia del siglo XXI denota una alarmante necesidad de dar respuesta porque las cifras de la última década van en aumento. Parece evidente que una alternativa a la necesaria institucionalización de estos adultos mayores pasa por un fortalecimiento de lazos sociales (programas de voluntariado, casas asistidas, etc.) porque la ausencia de respuesta conlleva un claro riesgo de sintomatología depresiva (Uribe \& Valderrama, 2007).

De todas las psicopatologías que puede manifestarse en esta etapa del ciclo vital, además de las más visualizadas como es el caso de las demencias, las relacionadas con los disturbios emocionales cobra una especial significación (Urbina, Flores, García, Torres \& Torrubias, 2007). La razón se basa en que, a la debilidad/dependencia creciente de esta etapa y la ausencia de lazos/apoyos socio-familiares, el proceso adaptativo requiere de claves espirituales de sentido para afrontar la propia muerte. Este cóctel puede debilitar aún más la fragilidad (autoestima, sentido vital, desesperanza, etc.) y, por tanto, la mayor vulnerabilidad en la depresión (Bermejo, 2016). Por esta razón es importante analizar el reto del proceso de envejecimiento y la realidad del adulto mayor desde esquemas explicativos nuevos. 
Por este motivo nos ha parecido relevante hacer una primera aproximación del modelo PAS (el Marco de Poder, Amenaza y Significado) al reto del escenario del envejecimiento con la ampliación del horizonte que la complejidad de esta nueva realidad demanda. Para contextualizar brevemente el enfoque conviene señalar tres aspectos fundamentales: el modelo PAS es una alternativa a los sistemas diagnósticos tradicionales (DSM-5 o CIE-11) en cuanto a criterios clasificatorios; supone una articulación de patrones que se pueden identificar en el sufrimiento humano; abre un abanico de actuaciones en los campos clínico o de los servicios sociales lo que conecta la realidad de desempeño de la persona en distintos escenarios (Johnstone \& Boyle, 2018).

Esta tripe caracterización consideramos que es de enorme utilidad para el abordaje del adulto mayor porque operativiza un camino que ayuda a construir narrativas personales y sociales más adaptativas. Así, el primero de los elementos del marco explicativo es el Poder, traducido en nuestro campo a la percepción de control/autocontrol para el desempeño. Del equilibrio o desajuste a esta primera variable se deriva la percepción de amenaza, es decir, cómo la adversidad de la edad (de salud, económicas o de otro tipo) son valoradas por el adulto mayor. Lo que inevitablemente pasa por la lupa del significado atribuido, interpretación que depende de claves personales pero también de los esteriotipos, y visiones que tiene la sociedad respecto a los mayores. Como resultado final del proceso se generan las respuestas que oscilan desde el afrontamiento activo hasta la agresión o el aislamiento.

A la luz del esquema expuesto, con la secuencia explicativa y los ingredientes que la componen, puede ser una guía heurística para explicar respuestas y soluciones a esta realidad del envejecimiento. Y que, como hemos señalado, deriva en soledad y riesgo de depresión por lo que se requiere un empoderamiento individual y social del adulto mayor, con esquemas más amplios que la categoría diagnósticas clásicas permitían. Y con esta justificación previa pasamos a delimitar esta unidad biopsicosocial y espiritual que necesita, prioritariamente, integrar claves de sentido.

\section{DELIMITACIÓN: LA RESPUESTA AL QUÉ DEL SENTIDO}

La justificación que se ha desarrollado en el epílogo anterior del modelo PAS tiene como último elemento explicativo la cuestión del significado. Y precisamente vamos a ampliar y delimitar este concepto desde el abanico de las respuestas que le compiten a nuestro ámbito disciplinar, es decir, desde el humanismo estudiando la naturaleza del adulto mayor en perspectiva científica. 
Por tanto, dejaremos fuera del debate las respuestas de la religión o las ciencias ocultas, por ser acercamiento que sobrepasa nuestro marco epistemológico.

Y los límites en los que pretendemos encuadrar el tema se basan en la teoría sobre la gerotranscendencia propuesta por Tornstam (1989) con conexiones evidentes con la perspectiva psicosocial del desarrollo humano formulada por Erikson (2000). Con estos dos elementos claros podemos entender esta etapa del ciclo vital del adulto mayor, pero hay que integrar las claves fundamentales de sentido, en cuanto a contenidos, que provienen de las aportaciones de la psicología humanista (Frankl, 2012) y de la corriente de la psicología positiva (Seligman, 2003).

Así, cualquier propuesta de intervención que pretenda mejorar la calidad de vida o la satisfacción vital, y por tanto prevenir el aislamiento, la soledad y la depresión, debe pasar por una clara articulación de tres niveles explicativos: individuales y sociales, cognitivas y emocionales, sentido y felicidad. Por esta razón justificamos el planteamiento siguiendo la lógica de los tres niveles señalados integrando a la persona en su contexto (teoría gerotrascendente de Tornston), proponiendo la superación de la crisis vital de este periodo (integridad vs desesperación en términos eriksonianos), y dotando de vías y contenidos a la búsqueda del sentido vital y la felicidad (planteamientos complementarios de Frankl y Seligman con el supersentido y la felicidad significativa respectivamente). Vamos a justificar, brevemente, la contribución de cada nivel explicativo al conjunto, así como la interdependencia entre ellos.

Convienen precisar que la pregunta por el significado en lo concreto y por el sentido de la vida en lo global, que nos estamos planteando se encontraría en la categoría de preguntas empíricas, es decir, se ciñen al mundo concreto de la experiencia de las personas, por lo que obviamos las formales (plano conceptual) y las filosóficas (plano racional). Por esta razón nos parece oportuno la formulación de la teoría de la gerotranscendencia desarrollada por Tornstam (1999) a partir de una ingente recogida de encuestas a adultos mayores. Los resultados de las mismas señalan como elementos claves de esta etapa las siguientes: un cambio de metaperspectiva (de una visión materialista del mundo a otra de carácter trascendente); aumento de la satisfacción vital (madurez y sabiduría); integración y superposición de edades (ruptura de los ejes temporales); reinterpretación del pasado con frecuentes reconciliaciones; ampliación explicativa de los límites científicos tradicionales; y cambios en las prioridades y escalas de valores (despreocupación por el cuerpo, entre otros). Hay que señalar que aunque la teoría de la gerotranscendencia presenta algunas limitaciones (Rajani \& Jawaid, 2015) es una guía para entender el envejecimiento humano en su etapa final. 
Resulta obvio que esta descripción no podría incluir las realidades de todos los adultos mayores, más teniendo en cuenta lo señalado sobre las tasas de soledad y depresión que aquejan a una parte importante de este colectivo. Por esta razón es necesario descender a un segundo nivel explicativo que nos ayude a comprender por qué en la realidad personal de un adulto mayor se pueden conseguir las cuotas de gerotranscendencia señalados por Tornstam o por el contrario tornaría a una situación de malestar o depresión. Y en la superación de esta última crisis vital, a la que Erikson (2000) denomina integridad vs desesperación, creemos que está la clave explicativa. La integridad es el resultado de asimilar la aceptación de los cambios y las experiencias vividas, donde la seguridad o la satisfacción son la base de la vida construida con sentido. El otro polo, la desesperación, resulta de la no aceptación que se manifiesta en un bucle de cuestiones pendientes hacia el pasado y un temor hacia el futuro por la proximidad de la muerte.

La integridad vital implica una provisión de sentido espiritual a la existencia con dos logros relevantes específicos de esta etapa como son la esperanza y la fe. Si la propuesta de la gerotranscendencia deviene de un planteamiento sociológico y los de las crisis vitales de uno psicosocial, parecería que con el tema de la espiritualidad nos abocamos hacia más cercano al filosófico. Conviene aclarar que la pregunta por el sentido es universal y, además, se acrecienta en circunstancias vitales adversas o cuando se plantean los límites de la propia vida como es el suicidio o la muerte. Por este motivo la cuestión no es sólo filosófica, sino que para la realidad del adulto mayor se torna en la necesidad de dar una respuesta práctica. Ni siquiera entraremos en la matización de si la vida tiene sentido por ser o se lo debemos buscar (sentido objetivo vs. sentido subjetivo), cuestión muy abordada por filósofos de una y otra corriente (Bruno y Spinoza como ejemplos de la primera corriente y Nietzsche, Husserl o Sartre de la segunda). Desde una perspectiva psicológica, que es la que nos compete, el tercer nivel explicativo se encuentra ampliamente articulado en la tradición de la Psicología Humanista y de la Psicología Positiva, para dotar de contenido y procedimientos a la respuesta pragmática del sentido. Como hemos desarrollado ampliamente en un número monográfico de esta misma revista $(\mathrm{Ca}-$ baco, 2018) las implicaciones del sentido vital a lo largo del desarrollo humano es enormemente relevante y, además, en campos educativos, clínicos o sociales. La continuidad señalada en las tradiciones de Frankl y Seligman se operativizan en los tres niveles que ambos proponen: ausencia de sentido, sentido y supersentido (Frankl) y vida placentera, vida comprometida y vida significativa (Seligman). El paralelismo entre ambas formulaciones, separadas en casi medio siglo en sus propuestas concretas, tienen grandes transversalidades en el planteamiento, por más que en sus títulos epistemológicos puedan resultar diferentes dada la propia evolución de la ciencia psicológica (Cabaco, 2014). 
Sea porque se produce una tendencia a la descentralización, como sugiere Tornstam, o por una mayor focalización en el polo de la integración, como apunta Erikson, lo relevante es que el centro de gravedad oscila en torno al sentido, sea en óptica frankliana o de felicidad seligmaniana. Y como acertadamente reflexionan Sánchez y Mochietti (2013) en lo que respecta al pensamiento del adulto mayor, lo veamos como sujeto epistémico o psíquico, es un ser en desarrollo y lleva implícito un movimiento de reestructuración y complejización con las consecuencias derivadas en las transformaciones internas (intra-psíquicas) y de las relaciones con los demás (intersubjetivas). Sin olvidar el importante papel de la espiritualidad en la tercera edad que como búsqueda de lo transcendente (creencias, sentimientos y acciones) contribuyen al sentido y tienen repercusiones psicológicas en el plano conductual, cognitivo y afectivo (San Martín, 2007). Considerar la biografía de la persona para facilitar el envejecimiento positivo y ayudar a que la integre en su narrativa personal es una obligación de los profesionales que trabajan en este campo. Y con este compromiso se explicita en el apartado siguiente una propuesta metodológica para conseguir resultados basados en la evidencia.

\section{METODOLOGÍA: LA RESPUESTA AL CÓMO INTERVENIR}

Operativizar una respuesta metodológica al problema planteado requiere una articulación que sea fiel a una visión comprehensiva del proceso de envejecimiento en tres niveles (Viel, 2019): como una fase del desarrollo (integrando todas las edades), como la elaboración de un duelo (aceptando las pérdidas) y como un proceso de adaptación (reajustando posibilidades y expectativas). Y siguiendo el discurso de Tornstam (2005) una intervención que contemple la perspectiva gerotrascendente debe integrar la voluntad de sentido o dimensión noética (Frankl, 2012). Para este psicólogo vienés los universales de sentido o valores existenciales son tres: valores de creación (donación), valores de actitud (aceptación) y valores vivenciales (acogida). Como hemos señalado en otros trabajos $($ Cabaco, 2014) puede establecerse un cierto paralelismo entre este nivel autotrascendente de Frankl con el plano de la vida significativa de Seligman (2003). En la propuesta que veníamos desarrollando desde hace algunos años (Barahona, Cabaco \& Urchaga, 2013a; Barahona, Cabaco, Urchaga, FernándezMateos \& Lancho, 2015) denominada cognición Instruccional Positiva (CIP), las vías experienciales representarían el camino de desarrollo personal siguiendo el modelo que se representa en la Figura 1: 


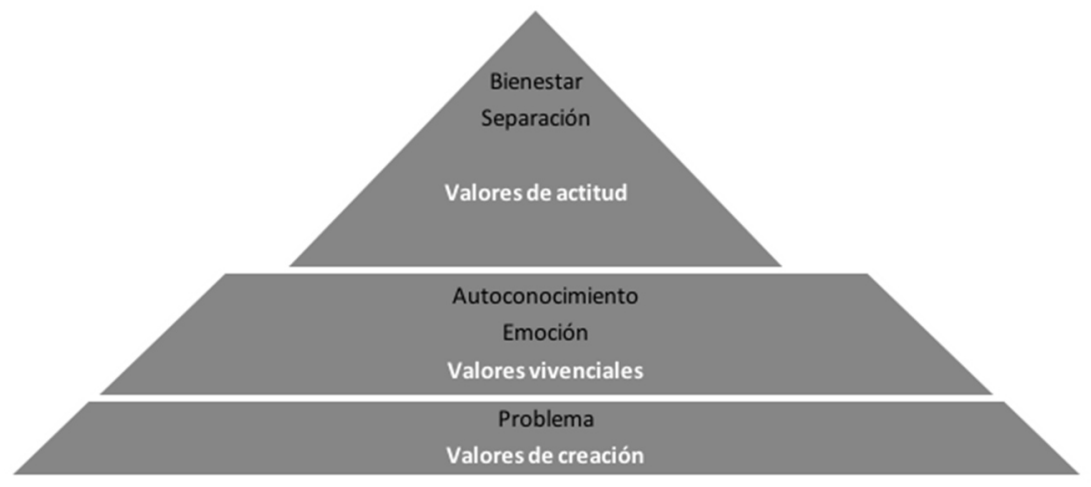

Figura 1: Niveles de la CIP y correspondencia con valores (elaboración propia)

La clave, por tanto, para superar la triada vital trágica (sufrimiento, culpa y muerte) pasa por una elaboración psicoeducativa del sufrimiento que es el objetivo del afrontamiento liberado que postula CIP. Estos cinco pasos o niveles abogan por un planteamiento ante el sufrimiento que pasa necesariamente por asumirlo (tomar postura ante el reto vital concreto), afrontarlo (asimilando, con sus tintes emocionales desde el propio yo) y trascenderlo (que implica el sentido). En definitiva, superar el sentimiento de inutilidad del sufrimiento en la radiografía de la CIP supone la consideración de los valores de creación (CIP 1 y 2 con la tarea a realizar), los valores de crecimiento interior (CIP-3 con la conciencia de los recursos propios) y los valores de madurez (CIP 4 y 5) en la libertad interior de salir del bucle de rencor y reorientar el proyecto vital hacia el bienestar y la felicidad (Barahona, Cabaco \& Urchaga, 2013b).

Desde el nuevo marco conceptual, para entender la realidad del sufrimiento en cualquier etapa del ciclo vital, y por supuesto ante el escenario del adulto mayor, las cuatro cuestiones básicas para reconstruir narrativas personales que ayuden a comprender la historia de vida de la persona serían, según Johnstone y Boyle (2018), las relativas al poder, la amenaza, el significado y las respuestas a ésta última. En primer lugar, la pregunta por el Poder ¿qué te ha pasado?, es decir, cómo ha actuado el poder en tu vida, qué cambios en la percepción de aumentos/pérdidas se han producido como consecuencia del envejecimiento. Es relevante contextualizarlo en la evolución experimentada en cuanto a declives o deterioros se hayan manifestado, y cómo la disminución de la autonomía personal está marcando una involución en la dimensión.

A continuación la pregunta por la Amenaza ¿qué tipo de amenaza representa para ti este cambio? Dado que el envejecimiento per se implica una involución de determinadas funciones personales (cognitivas y ejecutivas) o sociales (de 
tipo laboral y familiar) lo relevante es cómo este fenómeno es vivenciado por la persona. Dada la enorme heterogeneidad que se produce en los adultos mayores que coinciden con su edad cronológica, lo relevante es cómo interpretan y construyen sus trayectorias vitales.

La tercera cuestión clave es la pregunta del Significado ¿qué sentido le diste? Como patrón general de comportamiento evitamos o nos aproximamos a las situaciones/estímulos en función de lo que significan para nosotros. Cualquier pérdida de capacidad que sea percibida como amenaza puede inhibir conductas relacionales. Así, las limitaciones sensoriales (auditivas o visuales) que generen conciencia de amenaza pueden interpretarse como un elemento de incapacidad para participar en actividades con amigos o familiares, o, incluso, miedo a hacer el ridículo en esos contextos por la atribución del significado de incapacidad para un desempeño adecuado. A veces, con anticipación de consecuencias aversivas y con la consecuente presencia de malestar emocional.

Finalmente, la cuestión relacional con la respuesta a la Amenaza: ¿qué has hecho para afrontar la situación? Sabemos en clave motivacional que la tasa de alternativas de respuesta a cualquier situación vital disminuye a medida que desciende el número de refuerzos que se producen contigentemente a las conductas emitidas. Pero, además de la cantidad, interesa de forma especial la calidad de las mismas, su inserción en el patrón vital propio, la adecuación al nuevo momento vital o la innovación y creatividad que significa esta etapa del desarrollo.

Con estas cuatro claves podemos construir una primera radiografía vital para comprender la realidad del adulto mayor concreto que tenemos enfrente: qué significa y cómo funciona el poder es su vida (poder económico, interpersonal, ideológico, cultural, espiritual...); el tipo de amenazas que le son relevantes y su naturaleza (personal, familiar, social) y la que generan como sufrimiento mental; la traducción de estas amenazas en el significado personal atribuidas y los argumentos que soportan estas cosmovisiones; y las respuestas a la amenaza que son estrategias de supervivencia para afrontarlas.

Los autores citados señalan que debería completarse esta evaluación con dos cuestiones adicionales que son especialmente importantes a hora de afrontar el proceso de ayuda, desarrollo o reescritura. La primera cuestión adicional es la pregunta por las fortalezas, o más específicamente la accesibilidad a los recursos de poder que tiene la persona. En clave de Psicología Positiva el arsenal de fortalezas de la persona (Peterson \& Seligman, 2004) es un cambio esencial en lugar de centrarse en los déficits y limitaciones del modelo médico tradicional que adoptó la Psicología durante buena parte de su historia. Conocer estas 
fortalezas ayuda, además, a considerar los pilares sobre los que se tiene que construir el cambio.

Y como elemento globalizador final se plantearía la pregunta ¿Cuál es tu historia?, cuya finalidad es comprender la narrativa personal que constituye el propio guión vital. Entender lo que es relevante para él /ella, la repetición de episodios de éxito y fracaso, la atribución de significado a los mismos y cómo éstos se encuentran integradas o pendientes (bucle de rencor cíclico), serán las bases de la propia novela del éxito o del fracaso sobre el que trabajar. Y como herramienta fundamental, entre las alternativas de las denominadas terapias no farmacológicas (TNF), se encuentran la reminiscencia que ha demostrado una alta eficacia en evidenciar la disminución de sintomatología depresiva en adultos mayores (Afonso \& Bueno, 2010). De forma esquemática, siguiendo los tres niveles señalados al principio de este apartado vamos a ejemplificar el modelo de la CIP que hemos publicado recientemente (Cabaco, 2019).

Así, la reminiscencia optimiza el nivel de desarrollo, ayuda a la elaboración del duelo y facilita la adaptación porque es un mecanismo integrador de pasado y presente, y centrada en el recuerdo autobiográfico con la triple funcionalidad en el plano directivo, social e identitario. En esta obra se articulan los cinco niveles de la CIP, con propuestas de actividades en cada una de las dimensiones: reto, problemas, universo emocional, autoconocimiento, separación y bienestar. $\mathrm{Y}$ de forma monográfica se expone un programa centrado en la reminiscencia positiva (Rempos) con el objetivo adicional de generar un sesgo de recuerdos específicos positivos que faciliten la reexperimentación de situaciones agradables y placenteras, a la par que evite la sobregeneralización de las vivencias emocionales negativas.

\section{FINALIDAD: LA RESPUESTA AL PARA QUÉ DEL ENVEJECI- MIENTO CON SENTIDO}

Como conclusión a todo lo expuesto falta una breve reflexión acerca de la finalidad pretendida con este acercamiento a la realidad del adulto mayor intentando hacer visible el envejecimiento con sentido. Somos conscientes que añadimos más confusión a una selva conceptual donde no siempre están claros los límites entre definición de envejecimiento positivo, exitoso, saludable o activo. Hemos querido subrayar el apellido de sentido por ser el componente radical de la gerotranscendencia (Torstam, 2011).

Si el envejecimiento puede ser desarrollado con capacidad para elaborar los duelos y tener un carácter marcadamente adaptativo, debe pivotar en la búsqueda 
del sentido con una actitud personal para diseñar y vivir la última etapa del camino. Con el valor mayor que en otras etapas, la autodeterminación tiene que basarse en la libertad de elección en sus diferentes modalidades (interior, última y suprema). El miedo a la muerte, a la soledad, al abandono, a las pérdidas...debe sobreponerse a las respuestas de un sin sentido (huidas, defensa agresiva, sumisión o inmovilidad). Así lo evidencian formulaciones basadas en el riesgo y la descompensación que genera esta etapa del desarrollo humano (Gajardo, 2015).

Pero no se pueden cambiar actitudes individuales o sociales únicamente con planteamientos basados en un intencionalismo "buenista". Las críticas precisamente a la corriente del pensamiento positivo se basan en que únicamente son eslóganes teóricos que no sirven para modificar la realidad (Fernández Ríos \& Vilariño, 2016). Este acertado cuestionamiento hacia esas corrientes ideológicas no consideramos que se justifique en el caso de la Psicología Positiva que articula un discurso epistemológico y científico basado en la evidencia de resultados (Vázquez \& Hervás, 2008). Razón por la cual la respuesta al para qué tiene que ser operativa, visible y medible en términos de resultados empíricos.

En la línea argumental de Fierro (2008) entendemos que la psicología en sus intervenciones en general, y en el campo del envejecimiento en particular, debe comprometerse en favorecer en el adulto mayor mejor nivel de bienestar y felicidad. Particularmente hemos argumentado una tradición psicológica basada en el sentido, porque la situación de sufrimiento es quizás mayor. Específicamente como apuntan Freire y Fernández (2016) el bienestar psicológico de las personas mayores se explica por la primacía de factores como la autoaceptación, la autonomía la percepción de dominio de las demandas contextuales, las relaciones positivas con otras personas, las metas y proyectos vitales y el crecimiento personal.

A lo largo de estos últimos años hemos verificado empíricamente la eficacia de las intervenciones con las TNF en áreas cognitivas (Blazquez, Cabaco, Wobbeking, López \& Urchaga, 2018; Cabaco, Fernández-Mateos \& López, 2108) y emocionales (Cabaco, Fernández-Mateos, Crespo \& Schade, 2019) con el diseño de programas de estimulación del envejecimiento saludable en perspectiva tradicional con adultos mayores autónomos como institucionalizados (Wobbeking, Cabaco, Urchaga, Iglesias \& Camarón, 2016). También con resultados igualmente favorables en población afectada por algún tipo de patología frecuente en el proceso de envejecimiento como la diabetes mellitus tipo II (Lancho, Barahona, Fernández-Mateos, Cabaco, Sánchez \& Urchaga, 2017). Y no sólo en intervenciones grupales sino que en diseños de caso único se verifica igualmente su eficacia, innovando con la aplicación de las TICs (Sánchez, Cabaco, Urchaga y Villasan, 2019). 
Y como clave adicional, justificando la necesidad de que el proceso evolutivo del envejecimiento sea una responsabilidad en la que la población pueda responsabilizarse, con la noción de reserva cognitiva, desarrollada por Stern $(2002,2009)$, y que se ha evidenciado con un crecimiento creciente en la literatura científica (Tucker \& Stern, 2011; Carrasco, Barahona, Cabaco y FernándezMateos, 2018; Wöbbeking, Cabaco, Urchaga, Sitges \& Bonete, 2017). Porque su fortalecimiento disminuye la vulnerabilidad al deterioro y tenemos claves para identificar factores que contribuyen a su promoción, así como las estrategias de intervención que la fortalece (Cabaco, Fernández-Mateos, Villasan \& Carrasco, 2017). Además, dos tipos de reserva como la motivacional y la espiritual serán campos de investigación en las que evidenciar las fortalezas para una mejor calidad de vida en el adulto mayor.

Finalizamos resaltando la importancia de cambiar la ética de cuidados, muy representado en las declaraciones políticas de los organismos internacionales, con una ética de las necesidades y de los deseos del adulto mayor. Las aportaciones derivadas de la gerotrascendencia, creemos que son las que más se acercan a esta corriente y el modelo de intervención expuesto (CIP) es un camino para su consecución.

\section{REFERENCIAS BIBLIOGRÁFICAS}

Afonso, R. \& Bueno, B. (2010). Reminiscencia con distintos tipos de recuerdos autobiográficos: efectos sobre la reducción de la sintomatología depresiva en la vejez. Psicothema, 22(2), 213-220.

Barahona, M. N., Cabaco, A. \& Urchaga, J. D. (2013a). La Psicología Positiva aplicada a la educación: el programa CIP para la mejora de las competencias vitales en la Educación Superior. Revista de Formación e Innovación Educativa Universitaria, 6(4), 244-256

- (2013b). PIC program: implementation of the Positive Instructional Cognition in improving vital skills and quality of life. Atención Primaria, 45, 105-109.

Barahona, N., Cabaco, A.S., Urchaga, J.A., Fernández, L.M. \& Lancho, C.P. (2015). Experiencia educativa en España desarrollando la Cognición Instruccional Positiva. International Journal of Developmental and Educational Psychology, 1(1), 397-408.

Bermejo, J. C. (2016). La soledad en los mayores. Ars Medica Revista de Ciencias Médicas, 32(2), 126-144. doi: http://dx.doi.org/10.11565/arsmed.v32i2.264

Blazquez, A. Cabaco, AS, Wobbeking, M, López, A \& Urchaga, J.D. (2018). Estudio piloto de un programa de estimulación cognitiva en adultos mayores institucionalizados. Enseñanza e Investigación en Psicología, 23 (1), 58-66. 
Cabaco, A.S. (2014). Prefacio. En E. Salvino, De Frankl a Seligman: una apuesta por lo bueno y significativo de la vida (pp. 7-12). Brasilia (DF): Editorial Kirón

- (Coord.) (2016). Estimulación cognitiva del envejecimiento saludable. Salamanca: Publicaciones de la UPSA.

- (2018). Importancia del constructo sentido de la vida en diferentes ámbitos psicológicos aplicados. Cauriensia, 13, 17-38.

- (Coord.) (2019). Los niveles de la cognición instruccional positiva. Programa de reminiscencia CIP-CETYS para optimizar el bienestar de adultos mayores. México, Baja California: Publicaciones de CETYS-Universidad.

Cabaco, A.S. \& Fernandez-Mateos, L.M. (2014). Introducción: Las intervenciones intergeneracionales como terapias no farmacológicas para la prevención de la dependencia y la promoción de la autonomía personal. En A.S. Cabaco (dir.). Prismas intergeneracionales sobre la memoria autobiográfica: investigación y propuestas (pp. 5-17). Salamanca: Publicaciones de la UPSA.

Cabaco, AS, Fernández-Mateos, L. M, Crespo, A. \& Schade, N. (2019). Eficacia de un programa de estimulación de recuerdos emocionales positivos en adultos mayores del medio rural. Revista CienciAmérica, 8 (1), 15-32.

Cabaco, AS, Fernández-Mateos, LM \& Bañuelos, A. (2018). Mejora de habilidades mnésicas en el envejecimiento. Estudia Zamorensia, 17, 56-70.

Cabaco, AS, Fernández-Mateos, LM, Villasan, A. \& Carrasco, A. (2017). Envejecimiento activo y reserva cognitiva: Guía para la evaluación y la estimulación. Estudia Zamorensia, 16, 193-202.

Calero, D. \& Galiano, M.P. (2009). Utilidad de la evaluación de la plasticidad cognitiva en el diagnóstico diferencial del deterioro cognitivo y la pseudodemencia por depresión. Revista Española de Geriatría y Gerontología, 44(6), 323-330. Doi: https://doi.org/10.1016/j.regg.2009.06.012.

Carrasco, A., Barahona, N., Cabaco, A.S. \& Fernández-Mateos, L.M. (2018). E1 papel de la reserva cognitiva en el proceso de envejecimiento. Revista de Psicología, 19(1), 159-192.

Erikson, E. (2000). El ciclo vital completado. Buenos Aires: Paidós.

Fernández Ríos, L. \& Vilariño, M. (2016). Mitos de la Psicología Positiva: maniobras engañosas y pseudociencia. Papeles del Psicólogo, 37(2), 134-142.

Fierro, A. (2008). Conocimiento contra infelicidad: para una psicología epicúrea. Escritos de Psicología, 2(1), 7-23.

Frankl, V.E. (2012). Fundamentos y aplicaciones de la Logoterapia. Barcelona: Herder.

Freire, C \& Fernández, Ma . (2016). Calidad de vida y bienestar en la vejez. Madrid: Pirámide. 
Gajardo, J. (2015). Vejez y soledad: implicancias a partir de la construcción de la noción de riesgo. Acta Bioethica, 21(2), 199-205. doi: http://dx.doi.org/10.40 67/S1726-569X2015000200006.

Johnstone, L. \& Boyle, M. (2018). The Power Threat Meaning Framework: Overview. Leicester: British Psychological society.

Lancho, CP, Barahona, N., Fernández-Mateos, LM, Cabaco, AS, Sánchez, E. \& Urchaga, D. (2017). Entrenamiento de la memoria en personas mayores con diabetes mellitus tipo 2. Psychology, Society and Education, 9(3), 381-39. Doi 10.25115/psye.v9i3.860.

Mari, D. (2018). El camino de cien años. El arte de envejecer bien. Madrid: Biblioteca Nueva.

OMS (2015). Informe mundial sobre el envejecimiento y la salud. Luxemburgo: WHO.

Pérez-Rojo, G. \& Chulian, A. (2016). Cuenta conmigo, cuenta con ellos. Cuentos para la promoción del buen trato a las personas mayores. Madrid: COP.

Peterson, C. y Seligman, M.E. (2004). Character strenghs and virtues: a handbook and classifications. Oxford: Oxford University Press.

Rajani, F. \& Jawaid, H. (2015) Gerotranscendence: A Critical Review. Journal of Psychological and Clinical Psychiatry 4(1): 00184. DOI: 10.15406/jpcpy.20 15.04.00184.

San Martín C. (2007). Espiritualidad en la tercera edad. Psicodebate, 8, 111-127.

Sánchez, N., Cabaco, A.S., Urchaga, J.D. \& Villasan, A. (2019). Aplicación de técnicas de estimulación cognitiva en el envejecimiento utilizando TIC: estudio de caso. Neurama, Revista Electrónica de Psicogerontología, 6 (1), 4047.

Sánchez, M.L. \& Mochietti, A. (2013). Nuevos aportes para la comprensión del pensamiento en la vejez. Portal de Psicogerontología, 30, 15-29.

Seligman, M.E. (2003). La auténtica felicidad. Barcelona: Ediciones B.

Snowdon, D. (2003). Healthy aging and dementia: findings from the Nun Study. Annals of Internal Medicine, 139 (5), 450-454.

Stern, Y. (2002). What is cognitive reserve? Theory and research application of the reserve concept. Journal of the International Neuropsychological Society, 8(3), 448-460. Doi: https://doi.org/10.1017/S1355617702813248.

- (2009). Cognitive reserve. Neuropsychologia, 47(10), 2015-2028. Doi: 10.1016/j.neuropsychologia.2009.03.004.

Tornstam, L. (1989). Gero-trascendence: A reformulation of the disengagement theory. Aging, 1(1), 55-66.

- (1999). Gerotrascendence and the functions of reminiscence. Journal of Aging and Identity, 4(3), 155-166.

- L. (2000). Trasncendence in later life. Generations, 23(4), 10-14. 
- (2005). Gerotrascendence: A developmental theory of positive aging. New York, USA: Springer Publishing.

- (2011). Maturing into gerotranscedence. Journal of Transpersonal Psychology, 43(2), 166-180.

Tucker, A. M., \& Stern, Y. (2011). Cognitive reserve in aging. Current Alzheimer Research, 8(4), 354-360.

Urbina, J. R., Flores, J. M., García, M. P., Torres, L. \& Torrubias, R. M. (2007). Síntomas depresivos en personas mayores: prevalencia y factores asociados. Gaceta Sanitaria, 21(1), 37-42.

Uribe, A. F., Valderrama, L. \& López, S. (2007). Actitud y miedo ante la muerte en adultos mayores. Pensamiento Psicológico, 3(8), 109-120.

Vázquez, C. y Hervás, G. (2008). Psicología positiva aplicada. Bilbao: Desclee de Brower.

Viel, S. (2019). La experiencia emocional de envejecer. Temas de Psicoanálisis, $17,61-72$.

Wöbbeking, M, Cabaco, A.S., Urchaga; J.D., Sitges, E. \& Bonete, B. (2017) Reserva Cognitiva: Un análisis bibliométrico desde su implantación hasta la actualidad. Revista de Psicología de la Salud, 5(1), 86-113.

Wobbeking, M., Cabaco, A. S., Litago, D. U., Iglesias, A. \& Camarón, C. G. (2016). Intervención optimizadora del bienestar cognitivo y emocional en mayores institucionalizados: un estudio piloto. International Journal of Developmental and Educational Psychology, 1(2), 195-206. https://doi.org/ 10.17060/ijodaep.2015.n2.v1.335

\begin{tabular}{|c|c|}
\hline Antonio Sánchez Cabaco & Luz Ma Fernández Mateos \\
\hline Facultad de Psicología & Facultad de Educación \\
\hline Universidad Pontificia de Salamanca & Universidad Pontificia de Salamanca \\
\hline c/ Compañía, $n^{\circ} 5$ & c/ Compañía, $n^{\circ} 5$ \\
\hline 37002 Salamanca (España) & 37002 Salamanca (España) \\
\hline http://orcid.org/0000-0001-9953-5619 & http://orcid.org/0000-0003-2140-0890 \\
\hline
\end{tabular}

